\title{
Casuística de accidentes de motocicleta y lesiones en los alumnos de la Escuela de Tráfico de la Guardia Civil
}

\author{
Pacheco Delgado V. ${ }^{1}$, Arandojo Morales M. ${ }^{a} .^{2}$
}

Sanid. mil. 2013; 69 (2): 71-77; ISSN: 1887-8571

\begin{abstract}
RESUMEN
Objetivos: Conocer la causa de los accidentes de motocicleta y lesiones en los alumnos de la Escuela de Tráfico de la Guardia Civil, para establecer las medidas de protección y prevención más adecuadas. Material y métodos: La investigación se realiza sobre una muestra de 1.826 alumnos de Tráfico en la especialidad de Motorista, de ambos sexos, pertenecientes a nueve cursos, desde el 2009 al 2011.Para definir las lesiones se ha recurrido a la base de datos del Servicio de Sanidad del Centro, investigando un total de 277 Partes de Lesiones. Para conocer las causas, se analizan los Partes de Caída que se cumplimentan en las ambulancias. Resultados: Finalizan el Curso 1.437alumnos, causando baja 389. El porcentaje de lesionados por año es de $15,11 \%$ en 2009 , $15,45 \%$ en 2010 y $10,06 \%$ en 2011. El 2,3\% de los que causan baja del Curso, corresponde a lesiones graves durante las clases de conducción. El 28\% de los que causaron baja por falta de aptitud en la conducción, sufrieron algún tipo de lesión. Las zonas más afectadas son los miembros inferiores, superiores y tórax; en especial la pierna, tobillo, pie, rodilla y muñeca. Influyen en las causas de accidentes la edad, la climatología y el cansancio por el acumulo de horas de conducción. En cuanto al lugar donde se produce los accidentes, la pista número 5 «los peraltes» y la fase de campo (con motocicleta todo terreno), son más lesivas que la fase de carretera (con motocicleta de gran cilindrada); pero aquí, las lesiones son más graves. Hay instructores con un porcentaje de alumnos lesionados superior al del resto. Conclusiones: Existe una tendencia a una menor siniestralidad al disminuir el número de alumnos por Curso. Las contusiones, esguinces y fracturas son las lesiones más frecuentes y se localizaron principalmente en los miembros inferiores. Es preciso estudiar las causas del elevado número de accidentes en el obstáculo «los peraltes» y fase de campo. El instructor ejerce un papel primordial en la enseñanza del alumno y la prevención de los accidentes.
\end{abstract}

PALABRAS CLAVE: Accidente, Motocicleta, Lesión por accidente.

\section{Casuistry of motorcycle accidents and injuries in the Civil Guard's Traffic School}

SUMMARY: Objectives: to determine the cause of motorcycle accidents and injuries in the Civil Guard's Traffic School students, to establish more appropriate protective and preventive measures. Material and methods: The research was conducted on a sample of 1,826 students of both sexes in the Motorcyclist specialty, from nine courses, since 2009 to 2011 . To define the injuries we have resorted to the Center's Health Service database, evaluating a total of 277 Accident Reports. To determine the causes the Accident Reports which are completed in ambulances are studied. Results: 1.437 pupils finished the course, and 389 failed. Injuries percentage per year was $15.11 \%$ in $2009,15.45 \%$ in 2010 and $10.06 \%$ in 2011 . Of those who left the course $2.3 \%$ corresponds to serious injury during the driving lessons. $28 \%$ of those who left for lack of driving proficiency suffered injuries. Arms, legs and upper chest are the most affected areas, especially the leg, ankle, foot, knee and wrist. Age, weather and fatigue by the accumulation of driving hours influence the causes of accidents. With regards to the place where the accidents happen, the track number 5 «the cambers» and the field phase (with all terrain motorcycles), are more harmful than the road stage (with large cylinder motorcycle), but here the injuries are more severe. There are instructors with a higher percentage of students injured than the rest. Conclusions: there is a tendency to a lower number of accidents as the number of students per course is reduced. Bruises, sprains and fractures are the most common injuries and were located mainly in the lower limbs. It is necessary to study the causes of the high number of accidents in «the cambers» and the field stage. The instructor plays a vital role in teaching students and the prevention of accidents.

KEY WORDS: Accident, Motorcycle accident injury.

${ }^{1}$ Cap. Enfermero. Escuela de Tráfico de la Guardia Civil. Servicio de Sanidad. Mérida. Badajoz. España.

${ }^{2}$ Cap. Enfermera. Colegio de Guardias Jóvenes. Servicio de Sanidad. Valdemoro. Madrid. España.

Dirección para correspondencia: Valeriano Pacheco Delgado. Servicio de Sanidad. Escuela de Tráfico de la Guardia Civil. Duque de Ahumada s/n. 06800 Mérida. España. Tlfn: 924383348 Fax: 924370456

valerianopd@gmail.com

Recibido: 27 de noviembre de 2012

Aceptado: 5 de marzo de 2013

\section{INTRODUCCIÓN}

Accidente, se define etimológicamente como el suceso eventual o acción que altera el orden de las cosas y que, involuntariamente, origina daños en las personas u objetos, recibiendo el apellido de circulación o de tráfico cuando sobreviene en las vías públicas con ocasión del tránsito de vehículos ${ }^{1-4}$.

Para la Organización Mundial de la Salud (O.M.S.), los accidentes de circulación constituyen la principal causa de muerte en 
jóvenes con edades comprendidas entre los 15 y 30 años ${ }^{5}$. Según los datos que proporciona el Observador Nacional de Seguridad Vial de la Dirección General de Tráfico, en España en 2009, 2010 y 2011, fallecieron un total de 5.012 personas, de los que 763 fueron por accidente de motocicleta ${ }^{6}$. Resulta obvio que el número de heridos, en ambos casos, supera con creces al de muertos, representando un alto coste para la sociedad tanto a nivel económico como humano.

Los agentes integrantes de la Agrupación de Tráfico de la Guardia Civil, tienen como competencia la vigilancia del tráfico, la circulación, el transporte por carreteras y la seguridad vial en su conjunto ${ }^{7}$. Para ello, precisan, entre otros medios, de la utilización de coches patrulla y motocicletas de gran cilindrada. Están expuestos a diario a los múltiples peligros que genera la carretera, máxime cuando durante la conducción, no sólo atienden al pilotaje de su vehículo, sino que además están pendientes de las posibles infracciones y conducción del resto de usuarios ${ }^{8}$.

Durante los años 2009 al 2011, se produjeron un total de 1.795 accidentes en los agentes de tráfico, de los cuales, 578 fueron con víctimas y hubo un total de 12 muertos. La motocicleta fue el vehículo que estuvo implicado con mayor frecuencia en los accidentes con víctimas, con un total de 336; estos datos han sido facilitados por la Dirección Adjunta Operativa de la Agrupación de Tráfico.

En la Escuela de Tráfico de la Guardia Civil, en el periodo comprendido entre el año 2009 al 2011, se produjeron un total de 422 accidentes con lesiones, durante el desarrollo de las clases de conducción de motocicleta, según los datos de los partes de lesiones emitidos por el Servicio de Sanidad del Centro.

\section{Justificación del estudio}

Los trágicos datos avalan la importancia de investigar sobre las causas de los accidentes de motocicleta en la Escuela de Tráfico de la Guardia Civil al objeto de establecer las medidas preventivas o correctoras necesarias, para disminuir la siniestralidad entre en los alumnos motoristas, así como para aplicar estas soluciones al resto de los agentes de la Agrupación de Tráfico y demás motoristas de la red vial.

Es importante también, el estudio de las lesiones que se producen como consecuencia de los accidentes de motocicleta; conociéndolas, podremos buscar los elementos pasivos de seguridad adecuados para evitarlas o al menos minimizarlas.

No hay estudios anteriores que analicen la casuística de los accidentes de motocicleta y las lesiones en la Escuela de Tráfico de Guardia Civil. El Servicio de Sanidad del Centro, en sus memorias anuales, contabiliza las lesiones sufridas por los alumnos en los distintos cursos durante el año, sin dar importancia a las causas que las producen. La Dirección General de Tráfico informa a nivel estadístico, sobre la siniestralidad propia, abarcando las causas que pueden haber provocado los accidentes y la gravedad de los mismos. No estudia por tanto, la tipología de las lesiones, ni hace diferenciación de los accidentes acaecidos en la Escuela.

\section{Antecedentes y situación actual}

En 1844 el Duque de Ahumada fundó a la Guardia Civil, consciente de que el principal desafío del nuevo Cuerpo era la protección de caminos y viajeros ${ }^{9}$.
La construcción de carreteras y la aparición paulatina de automóviles y motocicletas cada vez en mayor número, hizo que la Guardia Civil creara el Parque Móvil en 1922. El primer «servicio de tráfico» tuvo lugar en 1926, a lo largo de 48 Kilómetros de la carretera Madrid - La Coruña. La II República y la Guerra Civil, apartó a la Guardia Civil de los cometidos de vigilancia de las carreteras españolas. En la década de los 40 se confió la vigilancia de éstas, a la Policía Armada. Posteriormente se encomendó a la Guardia Civil, instaurar un servicio motorizado en zonas concretas. Los resultados fueron más que aceptables, lo que favoreció la posterior creación de la Agrupación de Tráfico de la Guardia Civil en el año 1959.

El I Curso de Tráfico se desarrolló en el Centro de Instrucción (Madrid), convocándose a los primeros Oficiales y Suboficiales, produciéndose ya las primeras bajas por lesión en la conducción de motocicleta. Este personal instruyó al II curso de Circulación y Tráfico en la Academia Regional de El Escorial, para Sargentos, Cabos, Guardias y curiosamente «Practicantes de Enfermería» (Cabos o Guardias, con la misión de prestar los primeros auxilios en los accidentes de circulación). Se contó con 32 motocicletas marca Lube y 20 Sanglas. Durante las prácticas de conducción fallece un alumno, siendo el primer motorista de la Agrupación de Tráfico muerto en acto de servicio.

La Academia al no tener dependencias propias, vaga por distintas ubicaciones de la provincia de Madrid.

Desde el año 1962 hasta 1996, las prácticas de conducción se realizaron en unos terrenos de la zona militar de Campamento destinados a las maniobras de carros de combate, en Venta la Rubia (Madrid). En el año 1996 se traslada a Mérida (Badajoz) y adquiere la denominación de Escuela de Tráfico. Este Centro ya es específico para la obtención de la especialidad de Tráfico y cuenta con unas modernas instalaciones y con sus propias pistas de instrucción en la conducción de motocicletas ${ }^{10}$.

Las motocicletas también han evolucionado en el tiempo: desde las primeras y poco seguras Luben Ren y Sanglas 350, hasta las actuales BMW R 1200-RT dotadas con los más modernos sistemas de seguridad. Con respecto a la equipación del motorista, es importante destacar la evolución del casco como principal elemento de su seguridad: desde el casco Jet sin homologar (1959), hasta el actual System VI (2011, lleva incorporadas las transmisiones). Las prendas reflectantes pasaron de ser pequeñas bandas en el uniforme de servicio, a las actuales prendas, en las que prima el color reflectante. Estas prendas van provistas de protecciones en las zonas más vulnerables del cuerpo. Así mismo, el calzado actualmente es el adecuado para la práctica de la conducción de motocicleta, ya que hasta el año 2010, las botas utilizadas eran las mismas que las de equitación ${ }^{10,11}$.

Se ha evolucionado en mejoras de las pistas de conducción, en la dotación de modernas y más seguras motocicletas y en nuevos sistemas de seguridad pasiva. No obstante, como muestra este estudio, es preciso seguir progresando.

\section{Objetivos}

\section{General}

Conocer las causas de los accidentes de motocicleta y lesiones en los alumnos de la Escuela de Tráfico de la Guardia Civil. 
Casuística de accidentes de motocicleta y lesiones en los alumnos de la Escuela de Tráfico de la G.C.

Tabla1. Número de alumnos por curso.

\begin{tabular}{|c|c|c|c|c|c|c|c|c|c|}
\hline AñolPromoción & CXXXIV & CXXXV & CXXXVI & CXXXVII & CXXXVIII & CXXXIX & CXL & CXLI & CXLII \\
\hline 2009 & 300 & 277 & 250 & & & & & & \\
\hline 2010 & & & & 300 & 299 & 100 & & & \\
\hline 2011 & & & & & & & 100 & 100 & 100 \\
\hline
\end{tabular}

\section{Específicos}

Definir las lesiones que se producen en los accidentes especificando el tipo, localización y pronóstico de las mismas.

Estudiar la influencia de la motocicleta y la fase de conducción en el accidente con lesiones.

Estudiar el factor humano como desencadenante de los accidentes.

Concretar las medidas a adoptar para minimizar la siniestralidad durante las clases de conducción.

\section{MATERIAL Y MÉTODOS}

El estudio se realiza en la Academia de Tráfico de la Guardia Civil, en Mérida (Badajoz), sobre una muestra de 1.826 alumnos de Tráfico en la especialidad de Motorista, de ambos sexos y pertenecientes a los cursos CXXXIV, CXXXV y CXXXVI (año 2009), CXXXVII, CXXXVIII y CXXXIX (año 2010), y CXL, CXLI y CXLII (año 2011). Se decide descartar otros cursos de conducción de motocicletas realizados durante estos años, (Servicio de Protección de la Naturaleza (SEPRONA), curso de Dirección para Oficiales y cursos conducción para personal ajeno al Cuerpo), al objeto de mantener una homogeneidad en la muestra, dado que ni las fases de conducción, ni las horas lectivas, coinciden. Se decide igualmente ampliar el estudio a tres años para paliar en lo posible la diferencia numérica del año 2009 con 827 alumnos y del año 2011 con 300 alumnos.

Para definir las lesiones se han recopilado de la base de datos del Servicio de Sanidad de la Escuela de Tráfico, un total de 277 Partes de Lesiones. Se desechan 12 al comprobar que las lesiones se han producido por actividades ajenas a la conducción, quedando un total de 265. Se obtienen datos como: Diagnóstico (fractura, contusión, herida, quemadura, esguince, luxación, lesión muscular, otras lesiones); pronóstico, atendiendo al tiempo de recuperación, (leve de 0 a 15 días, menos grave de 15 a 30 días y grave más de 30 días); localización anatómica de las mismas (cabeza, cara-nariz-boca, cuello, tórax, abdomen, dorsal, lumbar, sacra, cadera-glúteo, hombro, brazo, codo, antebrazo, muñeca, mano, dedo mano, muslo, rodilla, pierna, tobillo, pie, dedo pie) y hemicuerpo lesionado.

Para estudiar la causa de los accidentes, se han valorado variables como Curso, fecha y hora del siniestro, fase de conducción, número y obstáculo de la pista, probable causa del accidente, motocicleta implicada e instructor responsable del alumno. Todos estos datos se extraen de los Partes de Caída que realizan los conductores de ambulancia y de las informaciones verbales efectuadas a los alumnos, en la oficina de Personal de la Escuela.

El número de alumnos por curso, los que finalizaron los mismos, las bajas por falta de aptitud en la conducción, las bajas definitivas por lesión, la edad y el número de horas lectivas, se obtienen de la base de datos de la Secretaría de Estudios. La fase de conducción en la que se produjeron los accidentes, del programa y base de datos del Grupo III (Departamento de Instrucción y Adiestramiento).

Los resultados se han recogido en una tabla elaborada con el programa Microsoft Office Excel 2010, por ser el que mejor se ajusta a nuestro estudio, donde se han calculado totales, porcentajes y medias.

\section{RESULTADOS}

El estudio se realiza sobre una muestra de 1.826 alumnos de Tráfico en la especialidad de Motorista, de ambos sexos. El $1,97 \%$ corresponde a mujeres y el $98,03 \%$ a hombres. Se decide no realizar comparaciones entre ambos, dada la diferencia numérica. No obstante resulta reseñable, que el 47,2\% de las alumnas no finalizan el curso por distintos motivos; el 41,6\% por falta de aptitud en la conducción y el 5,5\% por lesión grave durante la misma.

La muestra abarca los años 2009, 2010 y 2011. En cada año se han realizado tres cursos de tráfico, de tres meses de duración, en la modalidad de Motoristas. El número de alumnos por curso se distribuye como muestra la tabla 1 .

Finalizan el curso 1.437 alumnos, causando baja por distintos motivos 389. El 2,3\% de los que causan baja, corresponde a lesiones graves durante las clases de conducción. El 14,51\% del total de alumnos, resulta herido de diversa consideración (tabla 2).

Tabla2. Porcentaje de lesionados y de lesiones grave por año.

\begin{tabular}{|ccc|}
\hline & Lesionados \% & Lesiones graves \% \\
\hline $\mathbf{2 0 0 9}$ & $15^{\prime} 11$ & $2{ }^{\prime} 53$ \\
$\mathbf{2 0 1 0}$ & $15^{\prime} 45$ & $2{ }^{\prime} 14$ \\
$\mathbf{2 0 1 1}$ & $10^{\prime} 6$ & 2 \\
\hline
\end{tabular}

De los 175 alumnos que causan baja del curso por falta de aptitud en la conducción de motocicletas, el $28 \%$ sufre algún tipo de lesión. Porcentaje mayor al cómputo general de lesionados, que corresponde al 14,51\%.

Según el pronóstico de la lesión y teniendo en cuenta el tiempo de recuperación, obtenemos los siguientes resultados: Año 2009 (leve: 92, menos grave: 12, grave: 21); año 2010 (leve: 92, menos grave: 1, grave: 15) y año 2011 (leve: 26, menos grave: 0 , grave: 6).

Las lesiones producidas en orden de incidencia son: Contusión 142, Esguince 50, fractura 37, herida 24, lesión muscular 17, quemadura 11 y luxación 10. Tabla 3.

Según la localización anatómica: Pierna 37, tobillo 33, rodilla 28 , muñeca 27 , pie 25 , muslo 20 , tórax 19 , dedo mano 18 , 
Tabla 3. Frecuencia y ubicación de las lesiones.

\begin{tabular}{|c|c|c|c|c|c|c|c|c|}
\hline & Contusión & Esguince & Fractura & Herida & Les. muscular & Quemadura & Luxación & Total \\
\hline Pierna & 13 & & 6 & 5 & 4 & 9 & & 37 \\
\hline Tobillo & 8 & 24 & 1 & & & & & 33 \\
\hline Rodilla & 12 & 10 & 3 & 3 & & & & 28 \\
\hline Muñeca & 14 & 5 & 7 & & 1 & & & 27 \\
\hline Pie & 19 & 3 & 3 & & & & & 25 \\
\hline Muslo & 12 & & & 4 & 4 & & & 20 \\
\hline Tórax & 17 & & 1 & & 1 & & & 19 \\
\hline Dedo mano & 4 & 7 & 4 & 1 & & & 2 & 18 \\
\hline Mano & 13 & & 2 & 1 & & 1 & & 17 \\
\hline Hombro & 5 & & 5 & & & & 4 & 14 \\
\hline Codo & 2 & & 1 & 4 & & & 4 & 11 \\
\hline Dedo pie & 6 & 1 & 1 & & & & & 8 \\
\hline Brazo & 2 & & 2 & & 1 & 1 & & 6 \\
\hline Lumbar & 4 & & & & 2 & & & 6 \\
\hline Antebrazo & 1 & & 1 & 1 & 1 & & & 4 \\
\hline Cadera-glúteo & 2 & & & 2 & & & & 4 \\
\hline Cara-nariz-boca & 2 & & & 2 & & & & 4 \\
\hline Cuello & & & & & 3 & & & 3 \\
\hline Dorsal & 3 & & & & & & & 3 \\
\hline Sacro & 2 & & & 1 & & & & 3 \\
\hline Cabeza & 1 & & & & & & & 1 \\
\hline Abdomen & & & & & & & & 0 \\
\hline Total & 142 & 50 & 37 & 24 & 17 & 11 & 10 & 291 \\
\hline
\end{tabular}

mano 17 , hombro 14 , codo 11 , dedo pie 8 , brazo 6 , lumbar 6 , antebrazo 4, cadera-glúteo 4, cara-nariz-boca 4, cuello 3 , dorsal 3 , sacro 3 , cabeza 1 , abdomen 0 . Tabla 3 .

Se realiza un recuento de las lesiones, sin tener en cuenta la localización anatómica de las mismas, al objeto de determinar el hemicuerpo más afectado, obteniendo como resultado, 131 lesiones derechas y 133 lesiones izquierdas. En cambio, atendiendo a la ubicación de la lesión, resulta significativo: 15 lesiones de la muñeca derecha por 7 de la muñeca izquierda; 18 lesiones de la rodilla izquierda por 10 de la rodilla derecha; 18 lesiones en el pie izquierdo frente a 7 en el pie derecho.

Como posibles causas que influyen en los accidentes de motocicleta, se valoran:

a. Relación de las lesiones con el número de alumnos por curso: En el CXXXIV el porcentaje de lesionados es del 7\%, en el CXXXV del 19,5\%, en el CXXXVI del 20\%, en el CXXXVII del $13,3 \%$, en el CXXXVIII del $14,7 \%$, en el CXXXIX del $24 \%$, en el CXL del10\%, en el CXLI del $9 \%$ y en el CXLII del $13 \%$.

b. Edad: los alumnos pueden realizar el curso con edades comprendidas entre $21 \mathrm{y}$ 35 años, ambos inclusive. La media de edad de los alumnos de la muestra es de 28,5 años. La media de edad de los lesionados de 29,7 años.

c. Climatología: Se realiza un cómputo del número de le- sionados por mes. Enero 29, febrero 41, marzo 1; abril 46, mayo 60 , junio $1 ; 2^{\mathrm{a}}$ quincena septiembre 0 , octubre 78 , noviembre 8 y $1^{\mathrm{a}}$ quincena de diciembre 1 .

Los cursos se desarrollan en los trimestres enero-febreromarzo, abril-mayo-junio y segunda quincena de septiembre-octubre-noviembre-primera quincena de diciembre. Se comprueba que, los meses de octubre, mayo y abril son los de mayor índice de siniestralidad. El segundo trimestre con el 40,3\%, es donde más accidentes se produjeron. Destacar, que en los meses de enero-febrero, abril-mayo y primera quincena de septiembre-octubre, los alumnos se encuentran en la fase de pistas y de campo, mientras que en marzo, junio y segunda quincena de diciembre, los alumnos realizan las ultimas clases, de la fase de carretera.

d. Hora del accidente: Las clases de conducción se desarrollan de 08 a 14,10 horas, siendo el descanso de 10.15 a 10.45 horas (El horario reflejado en la figura 1, se aproxima al cuarto de hora, para una mejor simplificación en el estudio).

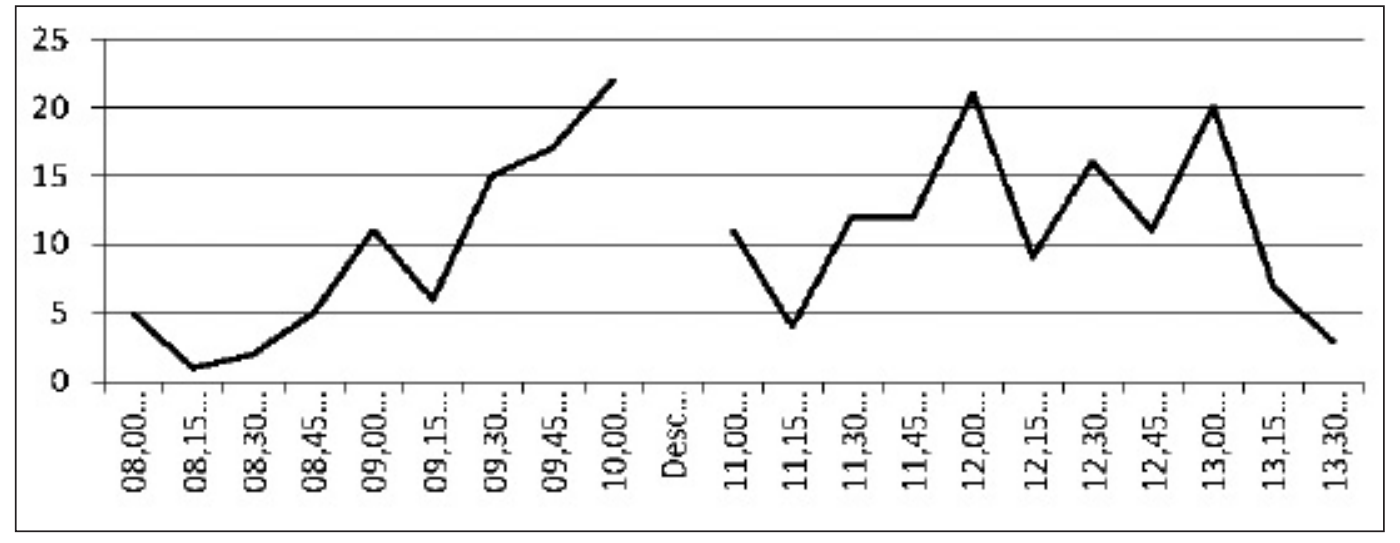

Figura 1. Relación del Número de heridos con la hora del accidente. 


\section{Casuística de accidentes de motocicleta y lesiones en los alumnos de la Escuela de Tráfico de la G.C.}

e. Fase de conducción: Se realizan tres fases al objeto de garantizar al alumno las habilidades, actitudes y conocimientos necesarios, para una conducción segura. La primera es la fase de pista, dividida a su vez en la fase de conos y fase de pistas de obstáculos; la segunda la fase de campo y la tercera la fase de carretera ${ }^{8}$.

Se han contabilizado en la fase de pista un total de 145 accidentes con lesiones, 89 en la fase de campo y 30 en la fase de carretera. Con respecto a las lesiones graves, el resultado se invierte: El porcentaje de lesiones graves en la fase de carretera es del $20 \%$, en la de campo del $15,7 \%$ y en la de pista del $15,1 \%$.

En la primera fase de pista (conos), se lesionan el 52,6\% frente al $47,4 \%$ de la $2^{\text {a }}$ fase de pista (obstáculos). La mayoría de las lesiones en la fase de pista (conos), se produjeron durante giros o frenadas con la rueda delantera durante la realización de ejercicios de riesgo (de pie sobre los estribos, de cuclillas sobre el sillín, de rodillas, sentado lateralmente, piernas sobre el manillar, tumbado adelante, piernas estiradas sobre el sillín, pie izquierdo sobre estribo derecho, sentado en manillar, y los ejercicios anteriormente citados sin una mano). Ningún ejercicio destaca del resto con respecto al número de caídas. En la fase de pista (obstáculos), las principales caídas se produjeron en los fosos, camellos y peraltes; cabe resaltar que en el obstáculo denominado «los peraltes» de la pista $\mathrm{n}^{\circ}$ 5 , se lesionaron el $43,2 \%$ de los alumnos de esta fase. Del total de las lesiones originadas en este obstáculo, el 21,8\% fueron de pronóstico grave.

Las principales causas de las caídas en la fase de campo, fueron debidas a las subidas o bajadas por desniveles pronunciados del terreno, por la introducción de la rueda delantera en roderas y socavones, o por pérdida de control de la motocicleta por piedras, arena o barro de los caminos.

En la fase de carretera y dentro de la Escuela, las principales causas de accidente se debieron a cambios de sentido haciendo conos, salida de vía en curvas y alcances entre motocicletas. Fuera de la escuela, la salida de vía fue la principal causa, seguida de colisiones con otros vehículos y problemas mecánicos.

f. Motocicleta: Son varios los modelos utilizados dependiendo de la fase de conducción. Para la primera fase, se utiliza la Honda CB 250 cc y la Yamaha SR 250 cc, en la fase de campo la Honda XR 250 R y en la fase de carretera la BMW R-850 o la BMW R-850 RT FL EVO 8 .

Se ha calculado la relación entre el número de accidentes con lesiones y la motocicleta implicada en el mismo, obteniendo en porcentajes: Honda CB $250(9,4 \%)$, Yamaha SR 250 $(45,2 \%)$, Honda XR 250 R $(33,9 \%)$ y BMW R-850 o la BMW R-850 RT FL EVO(11,3\%). Si se compara el modelo, con los accidentes de pronóstico grave, obtenemos el 20\% Honda CB 250, 20\% BMW R-850 o la BMW R-850 RT FL EVO, 15,5\% la Honda XR 250 R y el 14,1\% la Yamaha SR 250.

g. Instructor: Se relaciona a los lesionados con el instructor de conducción responsable de los mismos. Se aprecia, en base a los Partes de Caída, que ciertos instructores presentan un porcentaje mayor de alumnos lesionados: $(12,7 \%, 10,2 \%$, $9,18 \%, 7,14 \%, 6,12 \%, 5,61 \%, 5,10 \%, 4,08 \%, 3,57 \%, 3,06 \%$, $2,04 \%, 1,53 \%, 1,02 \%, 0,51 \%)$.

\section{DISCUSIÓN}

Los datos mostrados en esta investigación son objetivos y verificables, ya que han sido extraídos de documentos Oficiales de la Escuela de Tráfico de la Guardia Civil. Del mismo modo, la muestra de alumnos seleccionada es representativa, dado que se ha optado por cursos de conducción con idénticos programas de estudios y ampliándose a tres años, para paliar la diferencia numérica del año 2009 respecto al 2011.

La información que proporcionan los partes de lesiones, son absolutamente representativos de la lesividad de los alumnos durante las clases de conducción de motocicletas. Puede existir un mínimo error al no estar representadas todas las lesiones leves, ya que muchos alumnos las enmascaran y no acuden al servicio de sanidad, por el temor a ser dados de baja en el curso. Todas las lesiones importantes están reflejadas, ya que son atendidas in situ, por el personal de enfermería que realiza el servicio de ambulancia durante la clase de conducción.

Las causas de un accidente de circulación se deducen de los elementos que intervienen en el mismo: La vía, el vehículo y el hombre ${ }^{12}$. Los dos primeros son datos objetivables, mientras que los referidos al hombre, pueden ser representativos, pero en ningún caso objetivos (sueño, cansancio, perdida de concentración, problemas personales, enfermedad, medicamentos y falta de pericia entre otros).

Este estudio aporta datos interesantes, que pueden ayudar a disminuir la siniestralidad actual en las clases de conducción:

Con respecto a las lesiones, se ha demostrado que existe un porcentaje muy alto de lesionados y de lesiones graves, que es imprescindible reducir (14,55 \% y 2,3\% respectivamente). Es necesario buscar un equilibrio entre una óptima enseñanza de las técnicas de conducción y el menor número de heridos. El porcentaje del número de lesionados baja de forma considerable al disminuir el número de alumnos por año. Del 15, 11\% del año 2009 al 10,06\% del año 2011. La impericia en la conducción de motocicletas, juega un papel relevante en referencia al número de accidentes; de los 175 alumnos que causan baja del curso por falta de aptitud en la conducción de motocicletas, el 28\% sufre algún tipo de lesión, frente al 14,51\% del cómputo total. No se ha podido contabilizar, al no disponer de las calificaciones, la aptitud en la conducción de motocicletas de los alumnos que causaron baja por lesión grave. Todos los estudios consultados, confirman que la inexperiencia hace aumentar el riesgo de accidentes ${ }^{13-16}$.

Las lesiones más frecuentes son por orden de incidencia, las contusiones, esguinces, fracturas, heridas, lesiones musculares, quemaduras y luxaciones. La región anatómica más sensible es la pierna, seguida del tobillo, rodilla, muñeca, pie, muslo, tórax, dedo mano, mano, hombro y demás partes del cuerpo. Al igual que en el estudio realizado por la asociación de constructores europeos (ACEM) «Motorcicle accident in dephstudy» ${ }^{13}$, se confirma el miembro inferior como la región anatómica más vulnerable; tras éste, el miembro superior y tórax (parrilla costal). La mayoría de estas lesiones, podrían haberse evitado mejorando las protecciones con que actualmente realizan los alumnos las prácticas de conducción y en especial la de los miembros inferiores (rodilleraespinillera). El calzado utilizado hasta el momento, es la bota reglamentaria de campaña, que debe ser sustituida por un calzado 
adecuado. Para la fase de campo debe de dotarse al alumno de botas especiales con refuerzo en espinilla, tobillos y dedos.

Matizar que se han producido el doble de lesiones en la muñeca derecha que en la izquierda, quizás por la tendencia de apoyar en la caída la mano dominante. Sin embargo, las lesiones de la rodilla, pie y dedos del pie izquierdo, duplican a su homólogo derecho; pudiendo explicarse este hecho a que el motorista, aun siendo diestro, siempre tiende a apoyar primero la pierna izquierda (estas conclusiones han sido aportadas por los instructores del Centro).

Respecto a la causas de los accidentes: Referir que previo a la realización del Curso de Tráfico, todos los alumnos han superado un examen de selección, que incluye, además de unas pruebas de conocimiento, unas pruebas físicas y un reconocimiento médico y psicológico. No obstante, se ha comprobado que pese esta aptitud, la edad parece influir en las caídas y/o lesiones. La media de edad de los lesionados, supera a la media de edad del cómputo general. La elasticidad, fuerza, resistencia y reflejos disminuyen con ésta y en las clases de conducción se requiere de un alto nivel de exigencia física. En este caso, difiere de otros estudios, donde el riesgo de accidentes aumenta en motoristas con edades comprendidas entre los 18 y 25 años, disminuyendo en la franja de los 45-55 años ${ }^{13,15,16}$.

Se ha estudiado la fecha de la caída, buscando encontrar la influencia del clima en el accidente, obteniéndose resultados relevantes. Estos aumentan significativamente en los meses de octubre y mayo. El clima en Extremadura durante estos meses suele ser de temperatura agradable y ausencia de lluvia, condiciones óptimas para la práctica de la conducción de motocicletas. Coincide parcialmente, con un estudio realizado por la Mutua Madrileña y Línea Directa donde los meses de junio, julio, octubre y noviembre fueron los de más alta siniestralidad ${ }^{15,16}$. Con malas condiciones climatológicas, el alumno no emplea toda su capacidad, tendiendo a controlarse por miedo a las lesiones. El exceso de calor, tampoco ayuda a la realización de actividades donde se precise un gran esfuerzo físico.

Se realiza un estudio de la hora del accidente (figura 1), para determinar los periodos donde la accidentalidad es mayor. No se extraen conclusiones relevantes; antes del descanso de la clase de conducción los accidentes aumentan progresivamente; tras el descanso, se aprecian tres picos máximos a las 12, 12.30 y 13 horas, observándose posteriormente un descenso hasta el final de la clase (13.30 horas), que pudiera ser debido a la finalización de los ejercicios, recogida del material, puesta a punto del mismo y transmisión de novedades a los profesores de conducción. No obstante otros estudios, demuestran que la fatiga por el acúmulo de horas de conducción, entre otros factores, provoca pérdida de concentración y desgana, precipitando el accidente ${ }^{17}$.

En la fase de pista es donde se producen más lesiones en general, en especial en la pista de obstáculos $n^{\circ} 5$ «los peraltes»; sería necesario valorar si el nivel de pericia que proporciona, justifica el alto porcentaje de lesionados. Por número de sesiones, el porcentaje es mayor en la fase de campo, produciéndose el $90 \%$ de las caídas en las subidas o bajadas por cuestas pronunciadas. En la fase de carretera, en proporción, es donde más lesiones graves se originan. En esta fase dentro de la Escuela, las principales causas son el cambio de sentido en práctica con conos y la salida de vía en curva; fuera de esta, la principal causa es la salida de la vía en curvas y colisiones con otros vehículos. Esto difiere de otros estudios realizados en el ámbito civil, donde la principal causa del accidente es la colisión con otros vehículos ${ }^{14}$.

La BMW R-850 RT/FL EVO y la Honda CB 250, son las motocicletas que menos accidentes han causado, pero las que provocaron más lesionados graves (el 20\%); la primera lógicamente por el peso y la velocidad que puede alcanzar y la segunda, más que por sus características técnicas, por los ejercicios a veces muy complicados, que se desarrollan con ella. La Honda XR 250 utilizada para las prácticas en el campo, es la que más accidentabilidad ocasiona teniendo en cuenta las horas lectivas, ya que su manejo requiere de una técnica de conducción muy especial que difiere del resto de los modelos. La Yamaha SR 250 con el 45,2\%, posee el record de accidentes, pero al igual que la Honda influyen más los obstáculos de las distintas pistas, que la propia motocicleta.

Por último, cabe destacar el papel del profesor-instructor como un pilar clave en la prevención de los accidentes. Forzar en exceso a los alumnos, no proporcionar los descansos de forma adecuada, la no explicación correcta y progresiva de los ejercicios, enfrentar al alumno a un obstáculo de forma prematura, entre otras, son factores favorecedores de los accidentes. Sería importante tras los Cursos realizar reuniones, donde se evalué la siniestralidad y se busquen soluciones.

\section{CONCLUSIONES}

1. Existe tendencia a una menor siniestralidad al disminuir el número de alumnos por Curso.

2. Las contusiones, esguinces y fracturas son las lesiones más frecuentes en los accidentes de motocicleta. Estas se localizaron principalmente en los miembros inferiores.

3. La pista de obstáculo número 5 «los peraltes» y la fase de campo (en subidas o bajadas por desniveles pronunciados del terreno), son los mayores focos de producción de accidentes.

4. El instructor ejerce un papel primordial en la enseñanza del alumno y en la prevención de los accidentes.

\section{AGRADECIMIENTOS}

A mi Tutor y guía Cte. Enfermero D. Luis Orbañanos Peiro.

\section{BIBLIOGRAFÍA}

1. WIKIPEDIA: Accidente de tráfico [internet] [Acceso 5 de marzo 2012] Disponible en: http://es.wikipedia.org/wiki/accidente_de_tráfico

2. Accidentes de tráfico: Concepto de accidente; 2007. [internet] [acceso 5de marzo 2012] Disponible en: http://pantha-rei.blogspot.com.es/2007/01/unaccidente-es-un-suceso-o.html

3. Jesús Disla Álvarez: Que hacer ante un accidente de tráfico [internet] [acceso 5marzo 2012] Disponible en: http://www.ayuntamientoaltea.es/documentos/611.pdf

4. Seguridad pública: Accidentes de tráfico. Causas, clases y fases; 2009 [internet] [acceso 5 de marzo 2012] Disponible en : http://www.Seguridadpública. es/2009/03/accidentes-de-tráfico-causas-y-fases/

5. Dirección General de Tráfico. Educación y Seguridad Vial: tema 89. Los accidentes de circulación [internet] [acceso 8 marzo 2012] Disponible en: www.dgt.es 


\section{Casuística de accidentes de motocicleta y lesiones en los alumnos de la Escuela de Tráfico de la G.C.}

6. Dirección general de Tráfico: estadísticas e indicadores. Anuario estadístico general [internet] [acceso 8 marzo 2012] Disponible en: http://www.dgt.es/portal/es/seguridadvial/estadistica/publicaciones/anua riogeneral/

7. Guardia Civil. Seguridad Vial. Agrupación de Tráfico: Misión [internet] [acceso 9de marzo 2012] Disponible en: http://www.gurdiacivil.es/es/institucional/especialidades/Seguridadvial/in dex.html

8. Vidal Arana J.L. Conducción de motocicletas. Dirección General de la Policía y la Guardia Civil. Mérida. Escuela de Tráfico de la Guardia Civil; 2010 (91-5).

9. López Corral M, Rivas Gómez F, Villanueva Morales A, Jar Couselo G, Garrido Sánchez PM. La Guardia Civil Española. Secretaria General Técnica del Ministerio del Interior. Madrid: Asociación Pro huérfanos de la Guardia Civil; 1994 (26-2).

10. Agrupación de Tráfico de la Guardia Civil. 50 años Guardia Civil de Tráfico. Ministerio del Interior. Dirección General de Tráfico. Madrid: Ediciones San Mateo; 2009 (135-13).

11. VOROMV: Especial Guardia Civil de Tráfico; 2012 [internet] [acceso 16 de marzo 2012] Disponible en: http://www.voromv.com/especial-guardia-civilde-tráfico-1-de.html
12. Dirección General de Tráfico. Manual del conductor: Elementos que intervienen en los accidentes de circulación [internet] [acceso 17 marzo 2012] Disponible en: http://www.vialred.com/manual/34.pdf

13. Gstrail: Estudio sobre los accidentes de moto;2004 [internet] [acceso 30marzo 2012] Disponible en: http://www.gtrail.es/foro/accidentes-demoto-mayor-estudio-jamas-publicado-24660/ y http://www.maids-study. eu.php?error=hastolog

14. Motociclismo: Estudio sobre accidentes de moto; 2007 [internet] [acceso 30de marzo 2012] Disponible en: http://www.motociclismo.es/noticias/articulo/ estudio-sobre-accidente-moto-2501

15. Fundación Mutua Madrileña: I estudio de Siniestralidad vial en motocicletas; 2010 [internet] [acceso 5de abril 2012] Disponible en: http://www.fundaciónmutua.es/cs/

16. Línea Directa: Los accidentes más graves de moto.[internet] [acceso 5abril 2012] Disponible en: http://www.lineadirecta.com/sa_prensa/notas_prensa.nsf/ (vo02)/accidentes_moto\$FILE/accidentes_moto.pdf?open

17. RACE: la fatiga en la conducción. [internet] [acceso 15abril 2012] Disponible en: http://www.fatigayconduccion.com/estudios/informe_encuesta_fatiga08.pdf 